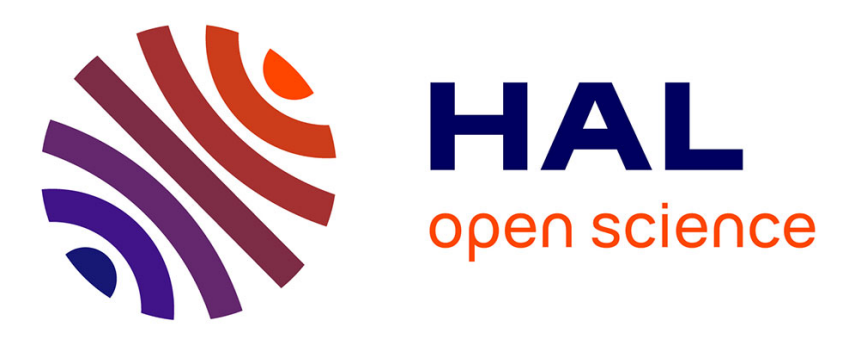

\title{
Wideband chaos from a laser diode with phase-conjugate feedback
}

Guillaume Bouchez, Chi-Hak Uy, Brice Macias, Delphine Wolfersberger, Marc Sciamanna

\section{- To cite this version:}

Guillaume Bouchez, Chi-Hak Uy, Brice Macias, Delphine Wolfersberger, Marc Sciamanna. Wideband chaos from a laser diode with phase-conjugate feedback. Optics Letters, 2019, 44 (4), pp.975. 10.1364/OL.44.000975 . hal-02447460

\section{HAL Id: hal-02447460 https://hal.science/hal-02447460}

Submitted on 21 Jan 2020

HAL is a multi-disciplinary open access archive for the deposit and dissemination of scientific research documents, whether they are published or not. The documents may come from teaching and research institutions in France or abroad, or from public or private research centers.
L'archive ouverte pluridisciplinaire HAL, est destinée au dépôt et à la diffusion de documents scientifiques de niveau recherche, publiés ou non, émanant des établissements d'enseignement et de recherche français ou étrangers, des laboratoires publics ou privés. 


\title{
Wideband chaos from a laser diode with phase-conjugate feedback
}

\author{
Guillaume BOUCHEZ ${ }^{1,2, *}$, Chi-Hak UY ${ }^{1,2}$, Brice MACIAS ${ }^{1,2}$, Delphine WOLFERSBERGER ${ }^{1,2}$ \\ and Marc SCIAMANNA ${ }^{1,2}$ \\ ${ }^{1}$ Chair in Photonics, LMOPS EA-4423 Laboratory, CentraleSupélec and Université Paris-Saclay, 2 rue Edouard Belin, Metz 57070, France \\ ${ }^{2}$ Chair in Photonics, LMOPS EA-4423 Laboratory, CentraleSupélec and Université Lorraine, 2 rue Edouard Belin, Metz 57070, France \\ "guillaume.bouchez@centralesupelec.fr
}

$\mathrm{W}$ e analyze experimentally and theoretically the chaotic dynamics generated by a laser diode subjected to phase-conjugate feedback. Phase-conjugation is obtained from four-wave mixing in a $\mathrm{BaTi}_{3} \mathrm{O}_{3}$ photorefractive crystal. We demonstrate that the chaos bandwidth first increases linearly with the feedback ratio but then saturates to relatively high values. A chaos bandwidth up to about $18 \mathrm{GHz}$ is achieved, which is more than five times larger than the free-running laser diode relaxation oscillation frequency. Numerical simulations confirm our experimental observations and unveil that the finite depth penetration into the crystal is responsible for the observed saturation.

Semiconductor lasers with feedback or injection are known to exhibit various non-linear dynamics, such as selfpulsation, quasi-periodicity and chaos $[1,2]$. The resulting laser chaos finds applications, in e.g. chaos-based cryptography [3], high-frequency random number generation [4] or chaotic lidar [5]. A crucial characteristic in those applications is the chaos bandwidth, which is generally defined as the span of frequencies of the chaotic signal $[6,7]$. The larger is the chaos bandwidth, the better is typically the system performance: random numbers can be generated at higher rate with wide-band chaos source [8], wideband chaos encrypted messages are harder to break [9], lidars with wide-band chaos source have better resolution [5]. In general the chaos obtained by a single laser diode with optical feedback has a bandwidth limited by the laser relaxation oscillation frequency [9]. To increase the chaos bandwidth beyond that limit, several sophisticated setups have been proposed. Experimentally, wideband chaotic semiconductor lasers have been achieved using optical feedback from two external cavities (16 GHz) [10], optical injection in a slave laser with feedback $(16 \mathrm{GHz}$ and $17 \mathrm{GHz}$ ) $[11,12]$, dual-wavelength injection into a laser with optical feedback (32 GHz) [13] or three lasers with optical feedback, injecting each others (35 GHz) [14]. Recently, replacing a conventional mirror by a phaseconjugate mirror, i.e. introducing the so-called phaseconjugate feedback (PCF) was found to significantly improve the chaos bandwidth of a laser diode [15]. Ref. [15] reports an enhancement of $30 \%$ of the chaos bandwidth thanks to PCF and reports of a chaos bandwidth of about $10.9 \mathrm{GHz}$ for a phase-conjugate mirror reflectivity of about $14 \%$, using SPS photorefractive crystal in a self-pumped ring cavity. Nonetheless, the detector bandwidth was limited to about $12 \mathrm{GHz}$. From Ref. [15] even larger values of chaos bandwidth can be expected for higher values of the phase-conjugate mirror reflectivity, hence justifying further analysis.

In this letter, we therefore extend our previous experiment on PCF-induced optical chaos. Using a PCF in a CAT configuration with a $\mathrm{BaTiO}_{3}$ crystal and not a selfpumped ring cavity with a $S P S$ crystal, we achieve a wideband chaos when increasing the reflectiviy of the mirror. Up to $18 \mathrm{GHz}$ chaos bandwidth is found for specific values of the feedback strength: this is more than five times larger 


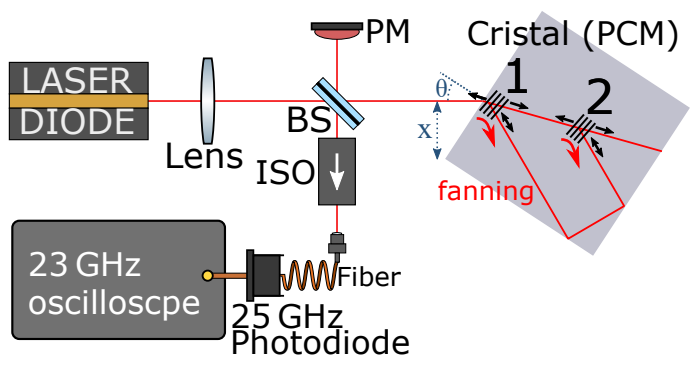

Figure 1: Experimental PCF setup using a CAT configuration.

than the free-running laser diode relaxation oscillation frequency. When the phase-conjugate mirror reflectivity is further increased, the chaos bandwidth saturates. A theoretical analysis confirms that the saturation of the chaos bandwidth originates from the finite penetration depth of the crystal, which induces a filtered phase-conjugate feedback.

The experimental setup is presented in Fig. 1. We use an edge-emitting semi-conductor laser diode. Its wavelength is $\lambda=852 \mathrm{~nm}$ and its injection current threshold is $J_{0}=14.9 \mathrm{~mA}$. The operating current ranges from $20 \mathrm{~mA}$ to $80 \mathrm{~mA}$. The free-running laser relaxation oscillation frequency is measured by analysis of the relative intensity noise and is equal to $3.9 \mathrm{GHz}$ at $50 \mathrm{~mA}$. To generate a phase-conjugate feedback (PCF), we use a phase-conjugate mirror (PCM) in the so-called CAT configuration [16]. The output laser beam is focused by a lens into a $R h$-doped $\mathrm{BaTiO}_{3}$ photorefractive crystal. The dimensions of this crystal are $5 \mathrm{~mm} \times 5 \mathrm{~mm} \times 5 \mathrm{~mm}$. The beam enters the crystal near the frontal edge. Due to the fanning, part of the light deviates from the straight propagation direction and this deviated beam is reflected by total internal reflection on the crystal sides. Four-wave mixing in zones 1 and 2 generates a backward wave which is the conjugate of the beam entering the crystal. A beam splitter (BS) (transmission of $79 \%$, reflection of $18 \%$ ) is placed to reflect a part of the incident beam into a fiber coupler. A Faraday isolator (ISO) is placed behind the coupler to avoid parasitic feedback into the laser. The multimode fiber is then connected to a $25 \mathrm{GHz}$ photodiode (PD), Newport 1414-50 High Speed Multimode Fiber-Optic Detector, whose electric signal is analyzed by an ultrafast oscilloscope (Tektronix DPO72304SX). The sampling rate of the oscilloscope is $50 \mathrm{GS} / \mathrm{s}$ while bandwidth of acquisition is $23 \mathrm{GHz} .18 \%$ of the backward beam is reflected and measured by a powermeter (PM), thus enabling to compute the feedback ratio, defined as the ratio of the free-running power of the laser over the power coming back into the laser.

The cavity is $53.5 \mathrm{~cm}$ long, so the round-trip time $\tau$ is $3.57 \mathrm{~ns}$ and the frequency associated with the external cavity is $1 / \tau$ i.e. $280 \mathrm{MHz}$. Two parameters are introduced to describe the geometry of the beam entering the PCM:
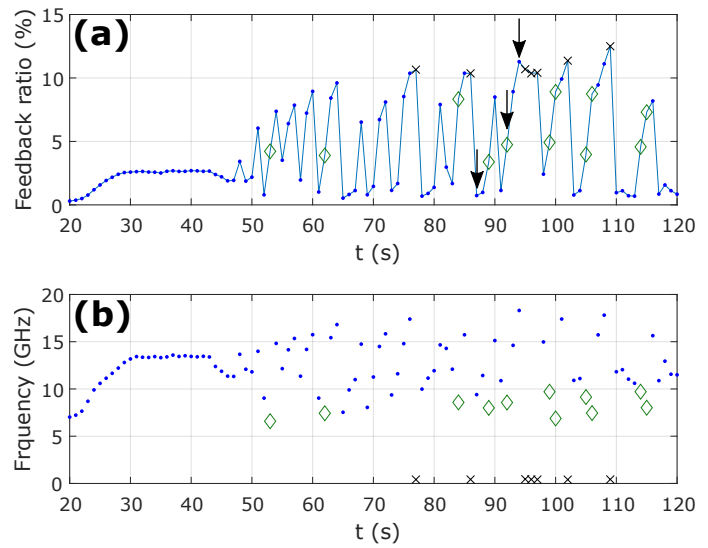

Figure 2: Evolution of (a) the feedback ratio and (b) the chaos bandwidth versus time. Blue dots are for chaotic dynamic states, green diamonds are for self-pulsing states and black crosses are for stationary states. For self-pulsing states (diamonds), the frequency is not the chaos bandwidth but the frequency of the main ECM.

$\theta$, the angle between the c-axis of the non-linear crystal and the input beam and $x$ the distance between the edge and the point of incidence in the crystal.

A laser diode with PCF is known to exhibit successive bifurcations of dynamic states, depending on the feedback ratio $\gamma$ : steady-state for zero or very weak optical feedback, self-pulsing dynamics at frequency near the relaxation oscillation frequency for low levels of feedback [17-20], chaos for higher values of feedback (>0.03\%) [18-20]. A further increase of the feedback strength results in a chaos crisis leading to self-pulsing $[19,20]$ and finally restabilization for very high values of the feedback strength $[17,18,20]$. Self-pulsing states are oscillating at multiples of the frequency of the external cavity and hence have been named ECMs or external-cavity modes [20]. The power spectrum of this external cavity laser output shows components at multiples of the external-cavity frequency. We chose to consider as self-pulsing dynamics all time series whose spectrum main energy is observed at discrete frequencies. Stationary time series are characterized by a signal AC spectrum equal to noise levels and chaotic states are characterized by broadband power spectrum encompassing many frequencies.

The reflectivity of a CAT-PCM is known to be varying against time on a slow time-scale as a result of the dynamics of the photorefractive induced gratings [21]. In Fig. 2 (a) we therefore follow the evolution of the PCM reflectivity versus time. For each reflectivity value, the laser exhibits therefore a specific dynamic state. The dynamics of the PCM is slow enough to allow us performing time series analysis. Each second, we record the achieved reflectivity value and simultaneously record and analyze a $2 \mu$ s-long power time-trace. The injection current is fixed at $50 \mathrm{~mA}$.

Depending on the observed dynamic, either the self- 

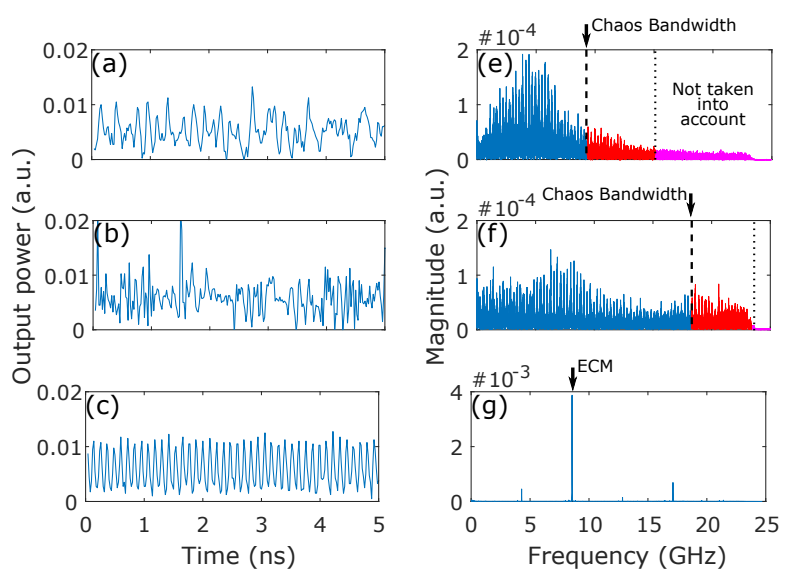

Figure 3: Time-traces of laser output power (left) and corresponding RF spectra (right) for different phase-conjugate feedback strengths. (a) has been recorded at $t=87 \mathrm{~s}(\gamma=0.46 \%)$, (b) at $t=94 s(\gamma=11.01 \%)$ and (c) at $t=92 s(\gamma=4.47 \%)$. The dashed line indicates the chaos bandwidth and the thinner dotted line indicates the frequencies on which chaos bandwidth was computed.

pulsing frequency (for regular self-pulsation) or the chaos bandwidth (for chaos) is reported in Fig. 2 (b). Chaos bandwidth is defined as the upper frequency of the RF spectrum that contains $80 \%$ of the signal linear energy [6]. To have snapshots of the RF spectrum of the signal, we use the Fourier transform of time series measured by the oscilloscope. Times series are $100 \mathrm{kS}$ long which is enough to study the high-frequency bandwidth. For that analysis we neglect frequency components whose power is close to the noise floor. More specifically, the frequencies from which the average energy of ten consecutive samples is $50 \%$ above the noise floor power level are not considered to compute the total energy.

For illustration, time series and RF spectrum obtained at different times (black arrows in Fig. 2) are plotted in Fig. 3. The RF spectrum of these time traces show frequency components at multiples of the cavity round-trip frequency $(280 \mathrm{MHz})$ since the dynamics originate from bifurcations on the ECM solutions. The chaos bandwidth is shown with an arrow. As mentioned before, the violet part of the spectrum is considered to be below the noise floor and is not taken into account for the chaos bandwidth analysis. At low feedback (i.e. $\gamma=0.46 \%$, see Fig. 3 (a)) the laser exhibits a chaotic behavior. The achieved chaos bandwidth is equal to $9.4 \mathrm{GHz}$ (Fig. 3 (e)) and is already larger than the relaxation oscillation frequency $(3.9 \mathrm{GHz})$. Fig. 3 (f) presents the RF spectrum at a higher feedback ratio $(\gamma=4.6 \%)$. The laser signal is still chaotic, but has a significantly larger chaos bandwidth, equal to $18,3 \mathrm{GHz}$. It is close to the measurement limit: as the oscilloscope has an analog bandwidth of $23 \mathrm{GHz}$, the limit chaos bandwidth will be around $18.4 \mathrm{GHz}(80 \%$ of $23 \mathrm{GHz}$ ). At a moderately large feedback ratio, the
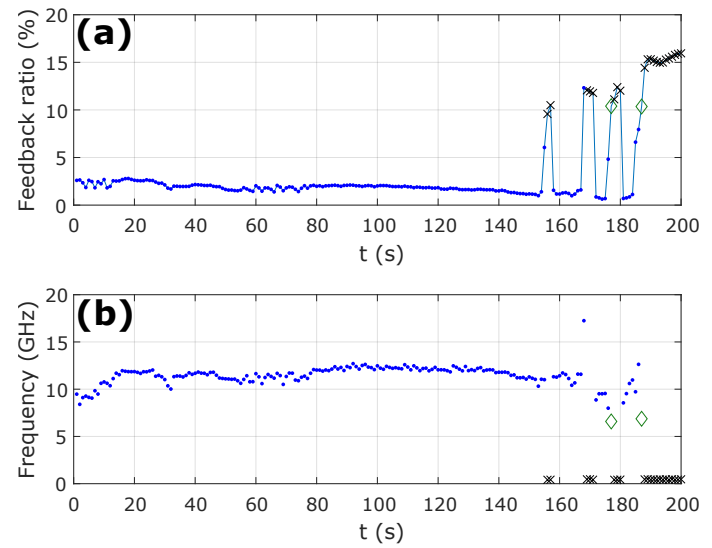

Figure 4: Evolution of (a) the feedback ratio and (b) the chaosbandwidth versus time when attempting to stabilize the reflectivity of the PCM. For regular self-pulsing states (diamonds), the frequency is not the chaos bandwidth but the frequency of the ECM.

system can also present self-pulsing ECMs. Figs. 3 (c) and (g) shows the system locked to an ECM at a frequency of $8.56 \mathrm{GHz}$ (around 31 times the frequency of the cavity) when $\gamma=4.47 \%$.

As the feedback ratio is not constant and is varying versus time, the system will not remain in the desired dynamical state for a long time. Some experiments to stabilize the reflectively of undoped $\mathrm{BaTiO}_{3}$ PCMs at visible wavelengths have been reported, but in experiments where an optical isolator prevents the laser to get destabilized by the PCF [22,23]. As suggested in Ref. [23], we scanned the behavior of crystal reflectivity when changing distance $x$ between the edge and the impact point. Whatever fixed angle $\theta$ is chosen, we do not find any long-term (over several tens of minutes) stable position for the PCM reflectivity, therefore contrasting with previous experiments at visible wavelengths and with stationary laser dynamics. Fig. 4 shows that a nearly stationary reflectivity can be achieved with similar external parameters. During more than two minutes, from $t=17 \mathrm{~s}$ to $t=147 \mathrm{~s}$, feedback ratio was stabilized around $4 \%$, and the laser diode remains chaotic with a bandwidth fluctuating between $10 \mathrm{GHz}$ and $12.7 \mathrm{GHz}$. However, at $t=145 \mathrm{~s}$, the reflectivity begins to oscillate, and then the variations of the PCM reflectivity destabilize the laser dynamics to either chaotic states with different bandwidths or even regular self-pulsating states or steady-states. Our systematic analysis of the PCM reflectivity variations over time and the resulting consequences on the laser diode dynamics therefore tells us that wideband chaotic dynamics with bandwidths up to five times the relaxation oscillation frequency can be stabilized over at least several minutes.

In Fig. 5 we summarize the evolution of either the chaos bandwidth (for chaotic states) or the main self-pulsating frequency (for ECMs) versus the feedback ratio, by concate- 


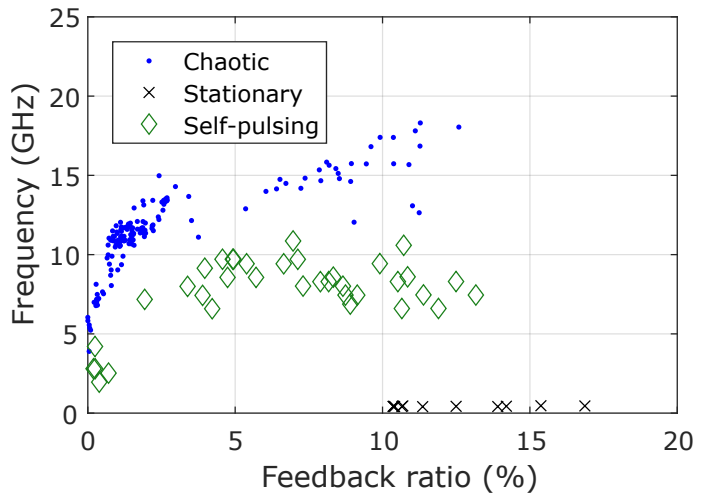

Figure 5: Experimental evolution of the chaos bandwidth versus the feedback ratio. For regular self-pulsing states (diamonds), the reported frequency is not the chaos bandwidth but the frequency of the ECM.

nating the data obtained during a $200 \mathrm{~s}$ long measurement. As the reflectivity of the PCM is varying continuously, there is some uncertainty in the measurement of the feedback ratio.

Still, that figure shows undoubtedly that chaos bandwidth shows a first rapid linear increase with the increase of the feedback ratio until reaching a value of about $15 \mathrm{GHz}$ at a feedback ratio of about $3 \%$. In a range of less than $3 \%$ of feedback ratio, the laser pulsating dynamics therefore increases its bandwidth by a factor 4 relatively to its free-running relaxation oscillation frequency. Then for moderately large feedback ratio, the chaos bandwidth shows a much slower increase with the feedback ratio and a maximum value of about $18 \mathrm{GHz}$ - i.e. close to the measurement limit - is measured at a feedback ratio of about $10 \%$. In that range of feedback ratios, the dynamics shows several stable ECMs at very high harmonic frequencies of the external cavity frequency, as also suggested by previous theoretical analyses [20]. For still larger feedback ratios, the laser diode re-stabilizes to a steady-state. It is also worth mentioning that, as again suggested by previous theoretical works, the dynamics of a laser diode with PCF shows significant multi-stability between chaotic attractors of different properties and even chaos and more regular self-pulsating solutions. This is also reflected in Fig. 5.

At first sight, the conclusions drawn from Fig. 5, i.e. a first rapid linear increase then almost saturation of chaos bandwidth with feedback ratio, contradict earlier theoretical predictions from our group: in Ref. [24] numerical simulations of a PCF laser model found a monotonic linear increase of the chaos bandwidth with the feedback ratio, as is also observed in a conventional optical feedback configuration but with a much faster increase. However, this simulation was performed on a PCF laser model with instantaneous phase-conjugation and therefore did not consider the filtering effect of the phase-conjugate mirror related to its finite penetration depth. In Refs. [20,25, 26] the PCF laser model has been extended to account for the finite penetration time $\tau_{r}$ of light inside the PCM.

In this paper, we simulate the filtered PCF laser model but more specifically analyzing the chaotic dynamics and their corresponding bandwidths. The model writes as [26]:

$$
\begin{gathered}
\dot{Y}=(1+i \alpha) Y Z+\kappa U \\
T \dot{Z}=P-Z-(1+2 Z)|Y|^{2} \\
\tau_{r} \dot{U}=Y^{*}(t-\tau)-U
\end{gathered}
$$

where $Y(t)$ is the complex normalized electric field of the laser, $U(t)$ is the complex normalized feedback field and $Z(t)$ is the real normalized carrier density. $t$ is the time, $\tau$ is the time delay, $\tau_{r}$ is the penetration time and $T$ is the electron lifetime. All these times are normalized by the photon lifetime $\tau_{p}=1.4$ ps. $\kappa$ is the dimensionless feedback strength, $\alpha$ is the linewidth enhancement factor and $P$ is the pump above threshold. We choose $\alpha=2$, $P=0.6016, T=1200$ and $\tau_{r}=50$ which are taken from our previous study of the same laser system [27]. Considering our experimental cavity round-trip length $(1.07 \mathrm{~m})$, we take $\tau=2548$.

We plot in Fig. 6 the chaos bandwidth versus the feedback rate. The model generates different dynamics: stable, self-pulsing and chaotic states. We analyzed the periodicity of the laser dynamics : if the laser output power is stationary, we consider the frequency to be equal to 0 (black crosses), if the output power is time-periodic, the displayed frequency is the main period (green diamonds) and if the signal is aperiodical, the frequency is its chaos bandwidth (blue dots). We also plot in red dotted line the evolution of the chaos bandwidth for a laser with a PCF with $\tau_{r}=1$ (instantaneous feedback). For $\kappa<0.015$, the chaos bandwidth is similar for filtered and unfiltered PCFs. From $\kappa=0.009$ to $\kappa=0.024$, the chaos bandwidth stays around $10 \mathrm{GHz}$. At higher feedback strengths, many high-frequency stable ECMs are found and for specific feedback rates the achieved chaos bandwidth is extremely high reaching about $18 \mathrm{GHz}$. The difference between the unfiltered PCF and the filtered PCF cases gets clear at large feedback rates: while the unfiltered PCF case predicts a monotonic linear increase of the chaos bandwidth with the feedback ratio, the filtered PCF case predicts saturation of the chaos bandwidth at large feedback rates. Furthermore the re-stabilization to the steady-state is only found theoretically in the filtered PCF case and not in the instantaneous PCF case, as already reported in Ref. [20]. The theory therefore matches qualitatively very well with the experimental findings and confirms the importance of accounting for the filtering properties of the PCM.

In summary, we report on the generation of wideband chaos from a laser diode with a single optical feedback. The achieved chaos bandwidth extends up to about five times the laser relaxation oscillation frequency. This 


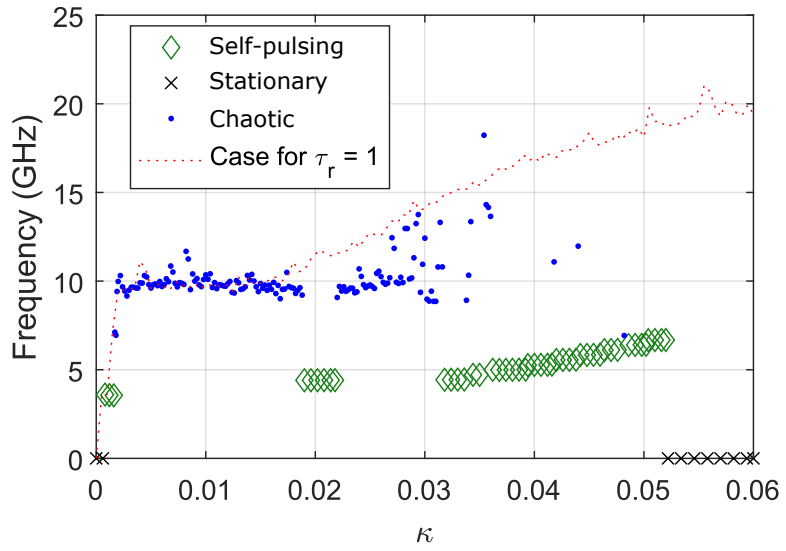

Figure 6: Theoretical variation of chaos-bandwidth (for chaotic state) and oscillation frequency (for self-pulsing states) of a laser with filtered PCF, versus the feedback rate. The red dotted line is the chaos bandwidth of an instantaneous PCF.

achievement is obtained thanks to the peculiar dynamical properties of phase conjugate optical feedback and does not require any additional feedback nor injection from a second laser. Interestingly and in contrast to earlier predictions, the chaos bandwidth increases in a non monotonic way with the increase of the feedback ratio. We demonstrate that this property results from the filtering property of the phase-conjugate mirror owing to its finite penetration depth.

Acknowledgements The authors thank the founders of the Chair in Photonics: Ministère de l'Enseignement Supérieur et de la Recherche, Région Grand-Est, Département Moselle, European Regional Development Fund (ERDF), Metz Métropole, Airbus GDI Simulation, CentraleSupélec and Fondation CentraleSupélec.

\section{References}

[1] V. Kovanis, A. Gavrielides, T. B. Simpson, and J. M. Liu, "Instabilities and chaos in optically injected semiconductor lasers," Applied Physics Letters, vol. 67, no. 19, pp. 2780-2782, 1995. [Online]. Available: https://aip.scitation.org/doi/10.1063/1.114591

[2] M. Sciamanna and K. A. Shore, "Physics and applications of laser diode chaos," Nature Photonics, vol. 9, no. 3, pp. 151-162, mar 2015. [Online]. Available: http://dx.doi.org/10.1038/nphoton.2014.326http: //www.nature.com/articles/nphoton.2014.326

[3] G. D. VanWiggeren and R. Roy, "Communication with Chaotic Lasers," Science, vol. 279, no. 5354, pp. 1198-2000, 1998. [Online]. Available: http:// science.sciencemag.org/content/279/5354/1198
[4] K. Hirano, K. Amano, A. Uchida, S. Naito, M. Inoue, S. Yoshimori, K. Yoshimura, and P. Davis, "Characteristics of Fast Physical Random Bit Generation Using Chaotic Semiconductor Lasers," IEEE Journal of Quantum Electronics, vol. 45, no. 11, pp. 1367-1379, 2009. [Online]. Available: https://ieeexplore.ieee.org/document/5291985

[5] F. Y. Lin and J. M. Liu, "Chaotic lidar," IEEE Journal on Selected Topics in Quantum Electronics, vol. 10, no. 5, pp. 991-997, 2004. [Online]. Available: https://ieeexplore.ieee.org/document/1366371

[6] _- "Nonlinear dynamical characteristics of an optically injected semiconductor laser subject to optoelectronic feedback," Optics Communications, vol. 221, no. 1-3, pp. 173-180, jun 2003. [Online]. Available: http://linkinghub.elsevier.com/retrieve/ pii/S0030401803014664

[7] F. Y. Lin, Y. K. Chao, and T. C. Wu, "Effective Bandwidths of Broadband Chaotic Signals," IEEE Journal of Quantum Electronics, vol. 48, no. 8, pp. 1010-1014, aug 2012. [Online]. Available: http://ieeexplore.ieee.org/document/6205601/

[8] N. Oliver, M. C. Soriano, D. W. Sukow, and I. Fischer, "Fast Random Bit Generation Using a Chaotic Laser: Approaching the Information Theoretic Limit," IEEE Journal of Quantum Electronics, vol. 49, no. 11, pp. 910-918, nov 2013. [Online]. Available: http://ieeexplore.ieee.org/document/6589109/

[9] A. Uchida, T. Heil, P. Davis, and T. Aida, "Highfrequency broad-band signal generation using a semiconductor laser with a chaotic optical injection," IEEE Journal of Quantum Electronics, vol. 39, no. 11, pp. 1462-1467, 2003. [Online]. Available: http://ieeexplore.ieee.org/lpdocs/ epic03/wrapper.htm?arnumber $=1242366$

[10] K. Schires, S. Gomez, A. Gallet, G.-h. Duan, and F. Grillot, "Passive Chaos Bandwidth Enhancement Under Dual-Optical Feedback with Hybrid III-V/Si DFB Laser," IEEE Journal of Selected Topics in Quantum Electronics, vol. 23, no. 6, pp. 1-9, nov 2017. [Online]. Available: http://ieeexplore.ieee. org/document/7994664/

[11] K. Hirano, T. Yamazaki, S. Morikatsu, H. Okumura, H. Aida, A. Uchida, S. Yoshimori, K. Yoshimura, T. Harayama, and P. Davis, "Fast random bit generation with bandwidth-enhanced chaos in semiconductor lasers," Optics Express, vol. 18, no. 6, p. 5512, mar 2010. [Online]. Available: https://www.osapublishing.org/oe/abstract. cfm?uri=oe-18-6-5512 
[12] A.-B. Wang, Y.-C. Wang, and J.-F. Wang, "Route to broadband chaos in a chaotic laser diode subject to optical injection," Optics Letters, vol. 34, no. 8, p. 1144, 2009. [Online]. Available: https://www.osapublishing.org/ol/abstract. cfm?uri $=$ ol-34-8-1144

[13] M. Zhang, T. Liu, P. Li, A. Wang, J. Zhang, and Y. Wang, "Generation of broadband chaotic laser using dual-wavelength optically injected FabryPérot laser diode with optical feedback," IEEE Photonics Technology Letters, vol. 23, no. 24, pp. 1872-1874, 2011. [Online]. Available: https: //ieeexplore.ieee.org/document/6032726

[14] R. Sakuraba, K. Iwakawa, K. Kanno, and A. Uchida, "Tb/s physical random bit generation with bandwidth-enhanced chaos in threecascaded semiconductor lasers," Optics Express, vol. 23, no. 2, p. 1470, jan 2015. [Online]. Available: https://www.osapublishing.org/abstract. cfm?URI=oe-23-2-1470

[15] É. Mercier, D. Wolfersberger, and M. Sciamanna, "High-frequency chaotic dynamics enabled by optical phase-conjugation," Scientific Reports, vol. 6, no. 1, p. 18988, may 2016. [Online]. Available: http://dx.doi.org/10.1038/srep18988http: //www.nature.com/articles/srep18988

[16] J. Feinberg, "Self-pumped, continuous-wave phase conjugator using internal reflection," Optics Letters, vol. 7, no. 10, p. 486, oct 1982. [Online]. Available: https://www.osapublishing.org/abstract. cfm?URI=ol-7-10-486

[17] A. Murakami and J. Ohtsubo, "Dynamics of Semiconductor Lasers with Optical Feedback from Photorefractive Phase Conjugate Mirror," Optical Review, vol. 6, no. 4, pp. 359-364, jul 1999. [Online]. Available: http://link.springer.com/10. 1007/s10043-999-0359-y

[18] J. S. Lawrence and D. M. Kane, "Contrasting conventional optical and phase-conjugate feedback in laser diodes," Physical Review A - Atomic, Molecular, and Optical Physics, vol. 63, no. 3, pp. 1-10, 2001. [Online]. Available: https://journals.aps.org/pra/ abstract/10.1103/PhysRevA.63.033805

[19] A. K. Dal Bosco, D. Wolfersberger, and M. Sciamanna, "Super-harmonic self-pulsations from a time-delayed phase-conjugate optical system," Applied Physics Letters, vol. 105, no. 8, p. 081101, aug 2014. [Online]. Available: http://aip.scitation.org/doi/10. 1063/1.4894119
[20] É. Mercier, L. Weicker, D. Wolfersberger, D. M. Kane, and M. Sciamanna, "High-order external cavity modes and restabilization of a laser diode subject to a phase-conjugate feedback," Optics Letters, vol. 42, no. 2, p. 306, jan 2017. [Online]. Available: https://www.osapublishing.org/abstract. $\mathrm{cfm}$ ?URI $=$ ol-42-2-306

[21] D. J. Gauthier, P. Narum, and R. W. Boyd, "Observation of deterministic chaos in a phase-conjugate mirror," Physical Review Letters, vol. 58, no. 16, pp. 1640-1643, apr 1987. [Online]. Available: https: //link.aps.org/doi/10.1103/PhysRevLett.58.1640

[22] T. Rauch, C. Denz, and T. Tschudi, "Analysis of irregular fluctuations in a self-pumped BaTiO3 phase-conjugate mirror," Optics Communications, vol. 88, no. 2-3, pp. 160-166, mar 1992. [Online]. Available: http://linkinghub.elsevier.com/retrieve/ pii/003040189290505L

[23] P. M. Jeffrey and R. W. Eason, "Lyapunov exponent analysis of irregular fluctuations in a self-pumped BaTiO3 phase-conjugate mirror, establishing transition to chaotic behavior," Journal of the Optical Society of America B, vol. 11, no. 3, p. 476, mar 1994. [Online]. Available: https://www.osapublishing. org/abstract.cfm?URI=josab-11-3-476

[24] D. Rontani, E. Mercier, D. Wolfersberger, and M. Sciamanna, "Enhanced complexity of optical chaos in a laser diode with phase-conjugate feedback," Optics Letters, vol. 41, no. 20, p. 4637, 2016. [Online]. Available: https://www.osapublishing. org/abstract.cfm?URI=ol-41-20-4637

[25] D. DeTienne, G. Gray, G. Agrawal, and D. Lenstra, "Semiconductor laser dynamics for feedback from a finite-penetration-depth phase-conjugate mirror," IEEE Journal of Quantum Electronics, vol. 33, no. 5, pp. 838-844, may 1997. [Online]. Available: http://ieeexplore.ieee.org/document/572159/

[26] L. Weicker, T. Erneux, D. Wolfersberger, and M. Sciamanna, "Laser diode nonlinear dynamics from a filtered phase-conjugate optical feedback," Physical Review E, vol. 92, no. 2, p. 022906, aug 2015. [Online]. Available: https://link.aps.org/doi/ 10.1103/PhysRevE.92.022906

[27] L. Weicker, C.-H. Uy, D. Wolfersberger, and M. Sciamanna, "Mapping of external cavity modes for a laser diode subject to phase-conjugate feedback," Chaos: An Interdisciplinary Journal of Nonlinear Science, vol. 27, no. 11, p. 114314, 2017. [Online]. Available: https://aip.scitation.org/doi/ 10.1063/1.5008392 